\title{
Competition between band and Mott insulator in the bilayer Hubbard model: a dynamical cluster approximation study
}

\author{
Hunpyo Lee ${ }^{2}$ * Yu-Zhong Zhang ${ }^{1 \dagger}$ Harald O. Jeschke ${ }^{2}$, and Roser Valentí2 \\ ${ }^{1}$ Shanghai Key Laboratory of Special Artificial Microstructure Materials and Technology, \\ School of Physics Science and Engineering, Tongji University, Shanghai 200092, P.R. China and \\ ${ }^{2}$ Institut für Theoretische Physik, Goethe-Universität Frankfurt, \\ Max-von-Laue-Straße 1, 60438 Frankfurt am Main, Germany
}

(Dated: September 5, 2018)

\begin{abstract}
We investigate the nature of the insulating phases in a bilayer Hubbard model with intralayer coupling $t$ and interlayer coupling $t_{\perp}$ at large interaction strength $U / t$ and half-filling. We consider a dynamical cluster approximation with a cluster size of $N_{c}=2 \times 4$, where short-range spatial fluctuations as well as on-site dynamical fluctuations are emphasized. By varying the band splitting $\left(t_{\perp} / t\right)$, we find that at $t_{\perp} / t \simeq 1.5$ the Mott behavior is rapidly suppressed in the momentum sectors $(\pi, 0)$ and $(0, \pi)$. At $t_{\perp} / t \simeq 2.5$ Mott features dominate in the momentum sectors $(\pi, \pi)$ of the bonding band and $(0,0)$ of the anti-bonding band and at $t_{\perp} / t \simeq 3.0$ a tiny scattering rate is observed in all momentum sectors at the Fermi level, indicating a transition from a Mott to a band insulator. We attribute such a momentum-dependent evolution of the insulating behavior to the competition and cooperation between short-range spatial fluctuations and interlayer coupling $t_{\perp}$ with the help of the Coulomb interaction $U$. Finally, we also discuss the possible appearance of non-Fermi liquid behavior away from half-filling.
\end{abstract}

PACS numbers: 71.10.Fd,71.27.+a,71.30.+h,71.10.Hf

\section{INTRODUCTION}

During the last decade intensive debates have centered around the question what happens when a system evolves from a band to a Mott insulator ${ }^{112}$ in the context of different models in different dimensions ${ }^{3}$ [15. like the extended Hubbard mode $e^{\sqrt[3]{6}}$ and the ionic Hubbard mode $e^{7} \frac{10}{10}$ in one dimension, or the ionic Hubbard mode 11$] 14$ in two dimensions. A model that has gained a lot of attention in recent years is the bilayer Hubbard model on a square lattice. The discovery of bilayer band splitting in angleresolved photoemission spectroscopy experiment $\$ 16$ for $\mathrm{YBa}_{2} \mathrm{Cu}_{3} \mathrm{O}_{6+x}(\mathrm{YBCO})^{17}$ suggested the consideration of such a model as a minimal model for describing doublelayered YBCO compounds.

In fact, the bilayer Hubbard model (see Fig. 1) was investigated by several groups within the dynamical mean field theory approximation (DMFT) $)^{18 / 19}$ and cellular DMFT 20. Transitions from metal to band insulator at small $U / t$ and from Mott to band insulator at large $U / t$ were reported with increasing $t_{\perp} / t$. However, by definition spatial fluctuations are completely ignored in DMFT $^{21}$ and such features like an intermediate nonFermi-liquid phase in the single-layered Hubbard model at half filling 224 are not captured by this approach. Moreover, only a small cluster size of $N_{c}=2 \times 2$ (two sites in one layer and two sites in another layer) was used in previous cellular DMFT calculations for the bilayer Hubbard mode ${ }^{20}$. For such sizes, the $C_{4}$ rotational symmetry of the square lattice is broken, resulting in an artificially enhanced local pair within each plane as the interaction $U / t$ is switched on. As reported in Ref. 25, such a choice is unable to describe an intermediate orbitalselective phase in a two-orbital Hubbard model. On the other hand, the dynamical cluster approximation (DCA) with clusters sizes of $N_{c}=2 \times 16$ (Ref. 26), the determinant quantum Monte Carlo method ${ }^{27}$ and the Gutzwiller approximation ${ }^{28}$-which do not suffer from the previous cluster limitations- were recently employed mainly to understand the nature of the superconducting state in the bilayer Hubbard model away from half filling. In view of the above results, and due to the limitations of the various approaches used, there is still not a full understanding of the transition from band to Mott insulating behavior as a function of $t_{\perp} / t$ in the bilayer Hubbard model at half filling.

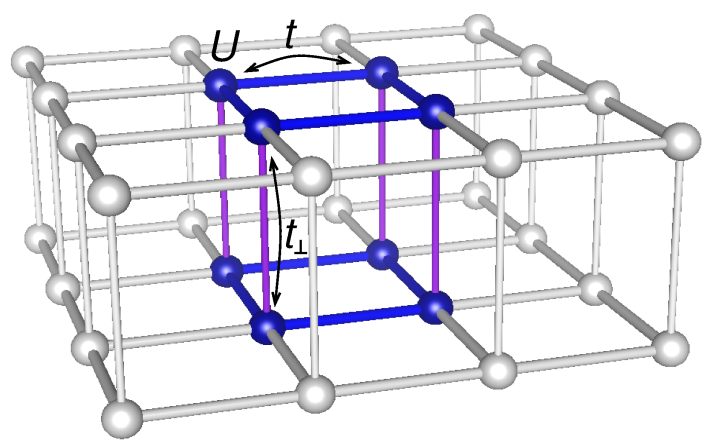

FIG. 1: (Color online) Cartoon of the bilayer Hubbard Hamiltonian. The $N_{c}=2 \times 4$ region studied here using DCA is shown in darker colors.

In the present work we concentrate on this question and study the bilayer Hubbard model at and away from half filling as the band splitting ratio $t_{\perp} / t$ is increased up to 4 at a large interaction strength of $U / t=6.0$ in the framework of the $\mathrm{DCA}^{29130}$. We consider one plaquette in each layer, i.e., a cluster size of $N_{c}=2 \times 4$, which 

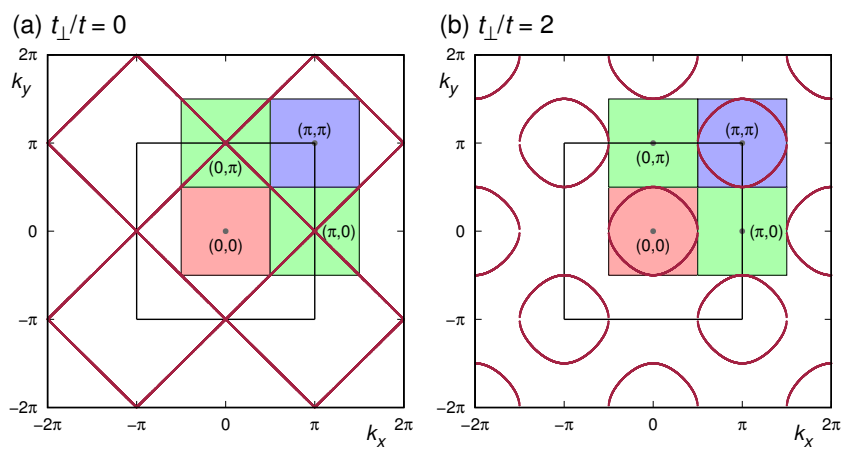

FIG. 2: (Color online) The Fermi surface for (a) $t_{\perp} / t=0.0$ and (b) 2.0 at half-filling in the weak coupling limit of $U / t=0$. The colored areas indicate the patches for momentum clusters $\mathrm{K}=(0,0),(\pi, 0),(0, \pi)$, and $(\pi, \pi)$ of the dynamical cluster approximation with $N_{c}=2 \times 4$.

keeps the rotation symmetry of the square lattice and allows for short-range spatial fluctuations. We focus on the lower temperature regime, which couldn't be accessed in previous studies $\frac{26}{2}$ due to the larger cluster sizes. We use an interaction-expansion continuous-time quantum Monte Carlo algorithm as an impurity solver $131 \sqrt[33]{33}$. Note that the value of the critical interaction strength for the metal-insulator transition is sensitive to the level of approximation considered 20135 . Within DCA and for the cluster sizes considered in this work, the value $U / t=6.0$ is deep in the insulating phase. In order to distinguish between a Mott and a band insulating regime we analyze the behavior of (i) the density of states near the Fermi level, (ii) the imaginary part of the self energy at the lowest Matsubara frequency and (iii) the momentum resolved electron density.

At small interlayer hopping $t_{\perp} / t$ we observe Mott insulating behavior in the DCA momentum sectors $(\pi, 0) /(0, \pi)$ and band insulating behavior in the DCA momentum sectors $(0,0) /(\pi, \pi)$ as was also obtained in cellular DMFT with $N_{c}=4$ in the single-band Hubbard mode $\mathrm{e}^{23}$. With increasing $t_{\perp} / t$ the Mott behavior is rapidly suppressed in the DCA momentum sectors $(\pi, 0)$ and $(0, \pi)$, and at $t_{\perp} / t \simeq 2.5$ it becomes dominant in the DCA momentum sectors $(\pi, \pi)$ of the bonding band and $(0,0)$ of the anti-bonding band. At $t_{\perp} / t \simeq 3.0$ a tiny scattering rate is observed in all momentum sectors at the Fermi level, indicating a transition from a Mott to a band insulator. Such a momentum-dependent evolution of the insulating state has not been reported in previous studies and will be analyzed in detail in the present work.

In particular, we find a monolayer plaquette singlet Mott insulator (m-PSMI) with strong intralayer plaquette order but weak interlayer antiferromagnetic (AF) correlations at small $t_{\perp} / t$ and a bilayer plaquette singlet Mott insulator (b-PSMI) with strong AF correlations between plaquettes belonging to different layers at intermediate values of $t_{\perp} / t$. At the critical value $t_{\perp} / t \simeq 3.0$ we observe a transition from a Mott to a band insulating state with tiny intralayer spin-spin correlations. Such a momentum-dependent phase behavior results from the competition and cooperation of short-range spatial fluctuations and interlayer coupling $t_{\perp}$ with the help of the Coulomb interaction $U$.

The paper is organized as follows. In Sec. III we present the model and dynamical cluster approximation. In Sec. III we present the density of states, the self-energy and electron density at each DCA momentum sector as well as spin-spin correlations and we discuss the nature of Mott insulator and band insulator at half-filling as well as non-Fermi liquid behavior away from half- filling. Finally, in Sec. IV we summarize our findings.

\section{MODEL AND METHOD}

The bilayer Hubbard Hamiltonian can be written as

$$
\begin{aligned}
H & =-\sum_{\langle i j\rangle m \sigma} t_{m}\left(c_{j m \sigma}^{+} c_{i m \sigma}+\text { h.c. }\right)-\mu \sum_{i m \sigma} n_{i m \sigma} \\
& -t_{\perp} \sum_{i \sigma}\left(c_{i 1 \sigma}^{+} c_{i 2 \sigma}+\text { h.c. }\right)+U \sum_{i m} n_{i m \uparrow} n_{i m \downarrow},
\end{aligned}
$$

where $c_{i m \sigma}\left(c_{i m \sigma}^{\dagger}\right)$ annihilates (creates) an electron with spin $\sigma$ at site $i$ and layer $m \in(1,2)$, and $\mu$ is the chemical potential. $t_{m}$ is the intralayer hopping matrix element between sites $i$ and $j$ in layer $m$ and $t_{\perp}$ is the interlayer hopping parameter which induces a band splitting into a bonding and an anti-bonding band. For $t_{m}=t(m=1,2)$ with $t$ as energy unit throughout this paper, the energy dispersion is given as $\epsilon^{\mathrm{A}, \mathrm{B}}(\mathrm{k})=\epsilon(\mathrm{k}) \pm t_{\perp}$, where $\epsilon(\mathrm{k})=$ $-2 t\left(\cos \mathrm{k}_{\mathrm{x}}+\cos \mathrm{k}_{\mathrm{y}}\right)$ and 'A' and 'B' indices denote antibonding (antisymmetric) $(+)$ and bonding (symmetric) $(-)$ states, respectively 18 .

The DCA is the cluster extension of single-site DMFT and the self-consistent equation can be written in momentum space with the assumption that the self-energy is constant in the Brillouin zone sectors that are considered. The cluster Green's functions are calculated by integration of each sector:

$$
\bar{G}_{\sigma}\left(\mathbf{K}, i \omega_{n}\right)=\frac{1}{N} \sum_{\tilde{\mathbf{K}}} \frac{1}{i \omega_{n}+\mu-\epsilon_{\mathbf{K}+\tilde{\mathbf{K}}}^{\mathrm{A}, \mathrm{B}}-\Sigma_{\sigma}\left(\mathbf{K}, i \omega_{n}\right)},
$$

where $N$ is the number of $\tilde{\mathbf{K}}$ points in each Brillouin zone sector, $\mu$ the chemical potential, $\mathbf{K}$ is the cluster momentum, $\epsilon_{\mathbf{K}+\tilde{\mathbf{K}}}^{\mathrm{A}, \mathrm{B}}$ is the dispersion relation for antibonding and bonding states, $\omega_{n}$ are the Fermionic Matsubara frequencies, and the summation over $\tilde{\mathbf{K}}$ is performed in each Brillouin zone sector. In our calculations, we considered a DCA cluster with $N_{c}=2 \times 4$, where $\mathbf{K}=(0,0)$, $(0, \pi),(\pi, 0)$ and $(\pi, \pi)$ (see Fig. 2). The converged selfenergy $\Sigma_{\sigma}\left(\mathbf{K}, i \omega_{n}\right)$ is evaluated by means of Eq. (2) and the Dyson equation and we employed the interaction expansion continuous-time quantum Monte Carlo approach as an impurity solver ${ }^{31} 33$. All calculations presented below are for a temperature $T / t=0.1$ and more than $5 \times 10^{6}$ 
QMC samplings are employed to measure the impurity Green's function.

In the non-interacting case $(U / t=0)$, the model shows a band insulating state at $t_{\perp} / t \geq 4$ due to the complete separation of bonding and anti-bonding bands, characterized by a formation of fully localized dimers between layers at half-filling.

\section{RESULTS}

\section{A. Half-filling}

In Fig. 3 we show the density of states $\rho(\omega)$ of the model at $T / t=0.1$ and $U / t=6.0$ for small, intermediate, and large band splittings with ratios $t_{\perp} / t=0.5,2.0$, and 4.0, respectively. At $t_{\perp} / t=0.5$ the gap is nonzero and dominated by the interaction $U$, and the bonding and antibonding states both contribute to the lower and upper Hubbard bands. On the other hand, at $t_{\perp} / t=4.0$, the gap, which is also significant, is dominated by the band splitting $2 t_{\perp}$. In this case, only the bonding state contributes to the band below the Fermi level while the antibonding one contributes to the band above the Fermi level. At intermediate $t_{\perp} / t=2.0$ the gap amplitude is reduced to a smaller value compared to the gap amplitudes at $t_{\perp} / t=0.5$ and 4.0. Here, both bonding and antibonding states significantly contribute to the bands above and below the Fermi level. However, the peak positions remain located at the same frequency as those for $t_{\perp} / t=0.5$ which indicates that Mott behavior is still present, i.e. the peak position is only dependent on $U / t$ and not on $t_{\perp} / t$.

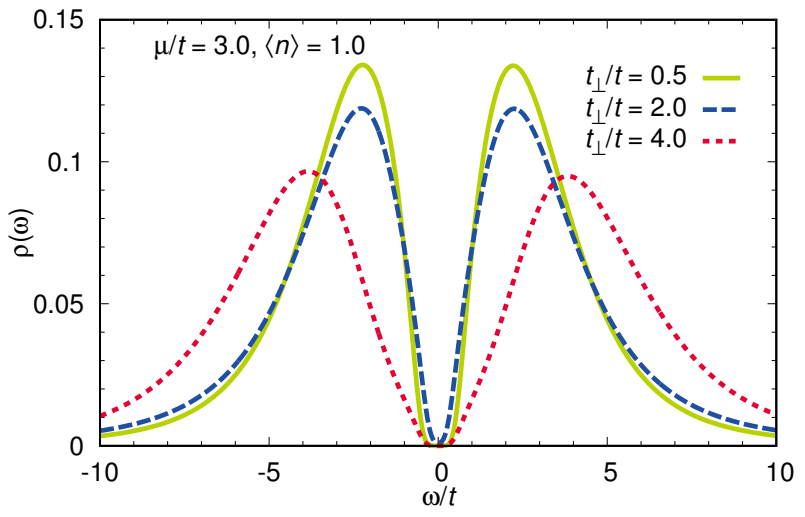

FIG. 3: (Color online) The density of states $\rho(\omega)$ for chemical potentials $\mu / t=3.0$ with half-filling at $U / t=6.0$ and $T / t=$ 0.1 . The band splitting is $t_{\perp} / t=0.5$ (solid line), 2.0 (dashed line), and 4.0 (dotted line). The Fermi level is at $\omega=0$.

In order to obtain a deeper insight into the nature of the phases as a function of the band splitting, we analyze in the following the cluster self-energy at the various DCA momentum sectors K. Figs. 4 (a) and (b) show the imaginary part of the DCA cluster self-energy
$\operatorname{Im} \Sigma\left(\mathbf{K}, \omega_{0}\right)$ at the lowest Matsubara frequency $\omega_{0}$ and the electron density $\langle n(\mathbf{K})\rangle$, respectively, as a function of $t_{\perp} / t$ for $U / t=6.0$ at half filling where $\mu / t=3.0$. Both quantities are shown for bonding (B) and antibonding (A) bands for the cluster momenta $\mathbf{K}=(0,0),(0, \pi)$ (identical to $(\pi, 0))$ and $(\pi, \pi)$

At small band splitting $t_{\perp} / t<0.5$, momentum selective Mott insulating phases with large scattering rates $\operatorname{Im} \Sigma\left(\mathbf{K}, \omega_{0}\right)$ are present in both bonding and antibonding bands at $\mathbf{K}=(\pi, 0)$ and $(0, \pi)$ momentum sectors where the electron density $\langle n(\mathbf{K})\rangle$ indicates almost halffilling, while the scattering rates at $\mathbf{K}=(0,0)$ and $(\pi, \pi)$ momentum sectors remain small suggesting a band insulating behavior with empty or fully filled electron density $\langle n(\mathbf{K})\rangle$ in these sectors. Further analysis on spin-spin correlations (Fig. 5) shows strong intralayer but weak interlayer antiferromagnetic correlations (Fig. 5 (a) and (b)). Moreover, the spin-spin correlation $\left\langle s_{i, m}^{z} s_{i+1, m^{\prime}}^{z}\right\rangle$ for different layers and nearest neighbor sites displays very weak ferromagnetic order at $t_{\perp} / t=0.5$ (see Fig. 5 (c)). We denote the state at small $t_{\perp} / t$ m-PSMI (monolayer plaquette singlet Mott insulator). This m-PSMI state has been reported to be present in the one-band Hubbard model investigated by a 4 -site cluster-DMFT approach 24 .

As $t_{\perp} / t$ increases, the scattering rates $\operatorname{Im} \Sigma\left(\mathbf{K}, \omega_{0}\right)$ at the $\mathbf{K}=(\pi, 0)$ and $(0, \pi)$ momentum sectors rapidly decrease towards zero while the scattering rate at the $(\pi, \pi)$ momentum sector of the bonding band (see $\Sigma_{B}(\mathbf{K}=$ $(\pi, \pi))$ in Fig. 4 (a)) and that at the $(0,0)$ momentum sector of the antibonding band (see $\Sigma_{A}(\mathbf{K}=(0,0))$ in Fig. 4 (a)) develop dramatically. In terms of the electron density $\langle n(\mathbf{K})\rangle$ in Fig. 4(b), the $(\pi, \pi)$ momentum sector of the bonding band and the $(0,0)$ momentum sector of the antibonding band are filled with about one electron at $t_{\perp} / t=2.2$. The strong scattering is caused by interactions between electrons in the $(\pi, \pi)$ momentum sector of the bonding band and the $(0,0)$ momentum sector of the antibonding band at $t_{\perp} / t=2.2$. The spin-spin correlations exhibit intermediate intralayer as well as interlayer antiferromagnetic correlations. Specifically, the ferromagnetic correlations for different layers and nearest neighbor sites reach a maximum in Fig. 5 (c). This means that the plaquette singlet orderings in each layer develop AF correlations. We denote this state bilayer plaquette singlet Mott insulator (b-PSMI).

When $t_{\perp} / t$ is further increased, tiny scattering rates $\operatorname{Im} \Sigma\left(\mathbf{K}, \omega_{0}\right)$ are observed in the $(\pi, \pi)$ momentum sector of the bonding band and the $(0,0)$ momentum sector of the antibonding band between $t_{\perp} / t=3.0$ and 4.0 , while the electron densities $\langle n(\mathbf{K})\rangle$ are not fully occupied (or empty) as shown in Figs. 4 (a) and (b). Such an insulator has been denominated a covalent band insulator 34 .

In the region $t_{\perp} / t>4.0$, all scattering rates disappear and the system is in a band insulating state, where the electron density $\langle n(\mathbf{K})\rangle$ are fully filled (or empty) in all momentum sectors and all bands. The ratio $t_{\perp} / t$ at which the Mott to band insulator phase transition hap- 

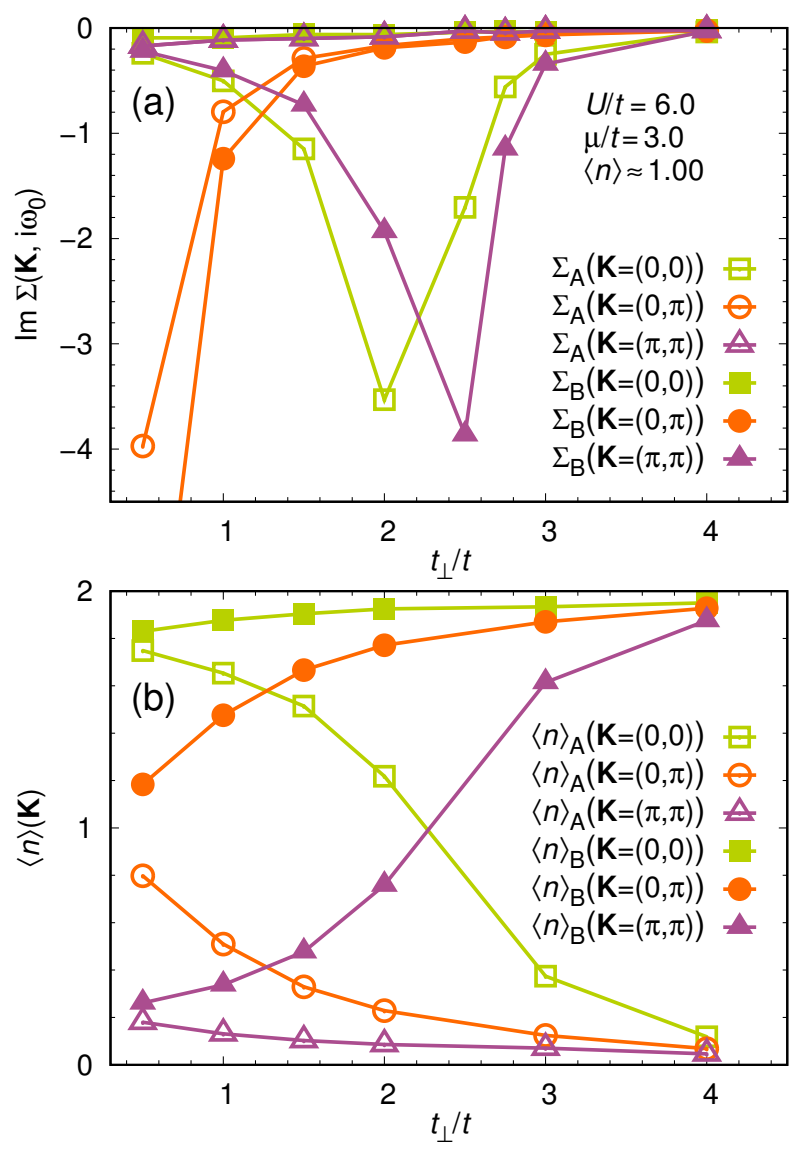

FIG. 4: (Color online) (a) Imaginary part of the DCA cluster self-energy $\operatorname{Im}\left(\Sigma\left(\mathbf{K}, \omega_{0}\right)\right)$ at the lowest Matsubara frequency $\omega_{0}$ and (b) electron density $\langle n(\mathrm{~K})\rangle$ as a function of $t_{\perp} / t$ at each DCA momentum sector K. Results are shown at a temperature $T / t=0.1$ and an interaction strength $U / t=6.0$ for chemical potential $\mu / t=3.0$ (half-filling $\left\langle n_{i}\right\rangle=1.00$ ). $\mathbf{K}$ are the DCA cluster momenta and indices A and B stand for antibonding and bonding, respectively.

pens is smaller than the the ratio for the metal-insulator transition in the noninteracting case $\left(t_{\perp} / t=4.0\right)$. This is due to the fact that strong correlation narrows the bandwidth of the bonding and antibonding bands and consequently a smaller band splitting is required for opening a band gap. The interlayer spin-spin correlations indicate a strong dimer state, while the remaining spin-spin correlations are extremely weak as shown in Fig. 5. The state at $t_{\perp} / t=4.0$ is a band insulator with isolated dimers between layers.

The momentum-dependent evolution of the insulating states can be well understood from the evolution of the Fermi surface in the weak coupling limit as a function of $t_{\perp}$. As shown in Fig. 2 (a), at $t_{\perp} / t=0$, all the Fermi surfaces are located in the momentum sectors $(\pi, 0)$ and $(0, \pi)$, indicating that poles determined by $\omega+\mu-\epsilon_{\mathbf{K}+\tilde{\mathbf{K}}}^{\mathrm{A}, \mathrm{B}}-\left.\operatorname{Re} \Sigma_{\sigma}(\mathbf{K}, \omega)\right|_{\omega=0}=0$ are only present in these two sectors. As the interaction $U$ becomes larger than the critical value of the Mott metal-to-insulator

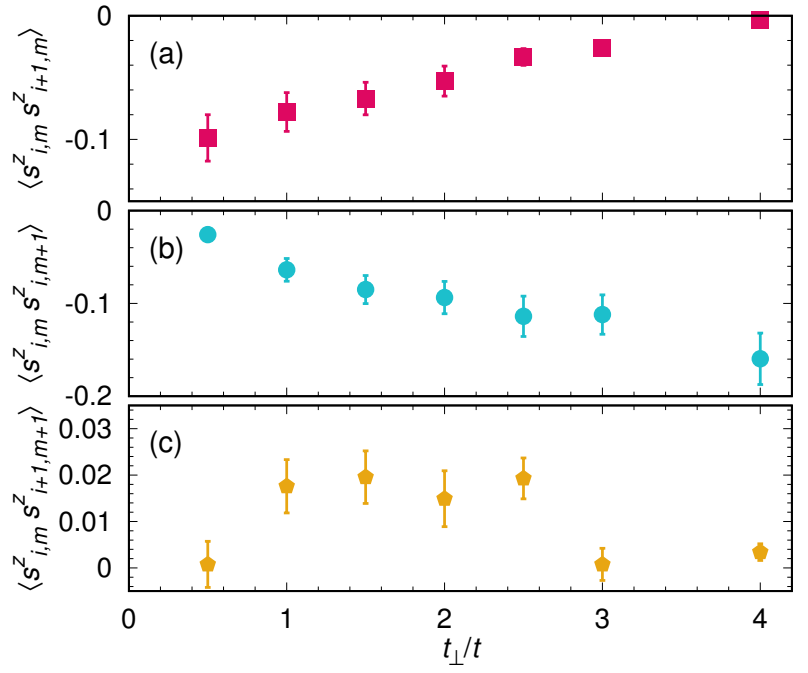

FIG. 5: (Color online) The spin-spin correlations $\left\langle s_{i, m}^{z} s_{i^{\prime}, m^{\prime}}^{z}\right\rangle$ as a function of $t_{\perp} / t$ (a) between $i$ and nearest neighbors site $i^{\prime}=i+1$ in the same layer $m$, (b) between layer $m$ and $m^{\prime}=m+1$ in the same site $i$, and (c) between $i$ and nearest neighbor site $i^{\prime}=i+1$ as well as layer $m$ and $m^{\prime}=m+1$ for $U / t=6.0$ and $T / t=0.1$.

transition, the opening of a gap at the Fermi level indicates $\operatorname{Im} G(\mathbf{K}, \omega=0) \rightarrow 0$, which requires large scattering rates at the positions of the poles. Therefore, Mott physics occurs in momentum sectors $(\pi, 0)$ and $(0, \pi)$. On the other hand, at $t_{\perp} / t=2.0$ (see Fig. 2 (b)), almost all the Fermi surfaces from the bonding band enter the momentum sector $(\pi, \pi)$ while those from anti-bonding band are mostly in the momentum sector $(0,0)$. In order to be a Mott insulator at large interaction $U$, large scattering rates are again inevitable in the momentum sectors $(\pi, \pi)$ of the bonding band and $(0,0)$ of the anti-bonding band since poles are now located in these sectors. Thus, the momentum-dependent evolution of the insulating states is a consequence of the evolution of the Fermi surface.

\section{B. Away from half-filling}

We concentrate in what follows on the origin of nonFermi liquid or pseudogap, which has been discussed extensively in the literature 36 , based on the bilayer Hubbard model away from half-filling. In the heavily doped case $(\mu / t=0.0)$, we observe at $t_{\perp} / t=0.5$ for $T / t=0.1$ a Fermi liquid like metallic behavior with $\operatorname{Im} \Sigma\left(\mathbf{K}, \omega_{0}\right)$ approaching small finite values due to the finite temperature effect (see Fig.6(a)). A quasiparticle peak is present at the Fermi level in the DOS (see Fig. 6 (c)). Close to the half-filled case, such as $\mu / t=2.0$ at $t_{\perp} / t=0.5$, large but finite scattering rates are observed in the antibonding $\mathbf{K}=(0, \pi)$ and the bonding $\mathbf{K}=(0, \pi)$ sectors due to the enhancement of intralayer short-range AF correlations. As a result, a pseudogap appears in the DOS at the Fermi level (see Fig. 6 (c)), reminiscent of the non- 

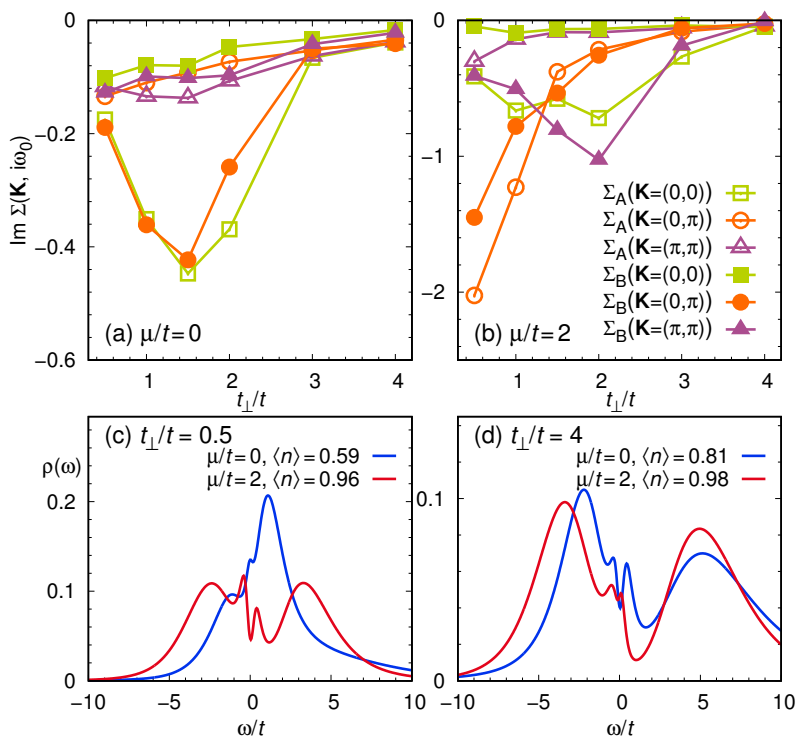

FIG. 6: (Color online) (a), (b) Imaginary part of the DCA cluster self-energy $\operatorname{Im}\left(\Sigma\left(\mathbf{K}, \omega_{0}\right)\right)$ at the lowest Matsubara frequency $\omega_{0}$ as a function of $t_{\perp} / t$. Results are shown at a temperature $T / t=0.1$ and an interaction strength $U / t=6.0$ for different values of the chemical potential: (a) $\mu / t=0.0$ (heavily doped case) and (b) 2.0 (close to half-filled case). The indices ' $K$ ' represent the DCA cluster momenta and 'A' and 'B' indicate antibonding and bonding, respectively. (c), (d) Density of states $\rho(\omega)$ for different chemical potentials $\mu / t$ at $U / t=6.0$ and $T / t=0.1$. The band splitting is (c) $t_{\perp} / t=0.5$ and (d) $t_{\perp} / t=4.0$. The Fermi level is at $\omega=0$.

Fermi liquid behavior observed in the single-band and multi-band Hubbard model $\sqrt{222} 25125141$. At $t_{\perp} / t=4.0$, though the scattering rates in all momentum sectors and all bands vanish due to the strong interlayer AF correlations (see Fig. 6 (b)), pseudogaps exist in both heavily doped and nearly half-filled cases, indicating that a strong scattering rate is not a necessary condition for the appearance of non-Fermi liquid behavior; rather the short-range AF correlations alone can be responsible for the non-Fermi liquid behavior.

Furthermore, in order to confirm the transitions from the Fermi liquid to Mott insulator via non-Fermi liquid as a function of electron doping (or chemical potential), we plot the $\operatorname{Im} \Sigma\left(i \omega_{n}\right)$ at the lower temperature $T / t=0.05$ and $t_{\perp} / t=0.5$ for $U / t=6.0$ in Fig. 7. In the case of $\langle n\rangle=0.60, \operatorname{Im} \Sigma\left(\mathrm{i} \omega_{0}\right)$ converges to almost zero indicating a Fermi-liquid behavior. As the electron density $\langle n\rangle$ approaches half-filling between $\langle n\rangle=0.87$ and 0.96 , $\operatorname{Im} \Sigma\left(\mathrm{i} \omega_{0}\right)$ converges to finite values. Such a behavior is typical for non-Fermi liquid states. Finally, at halffilling with $\langle n\rangle=1.00$ the system is a Mott insulator with $\operatorname{Im} \Sigma\left(\mathrm{i} \omega_{0}\right)$ exhibiting a diverging behavior.

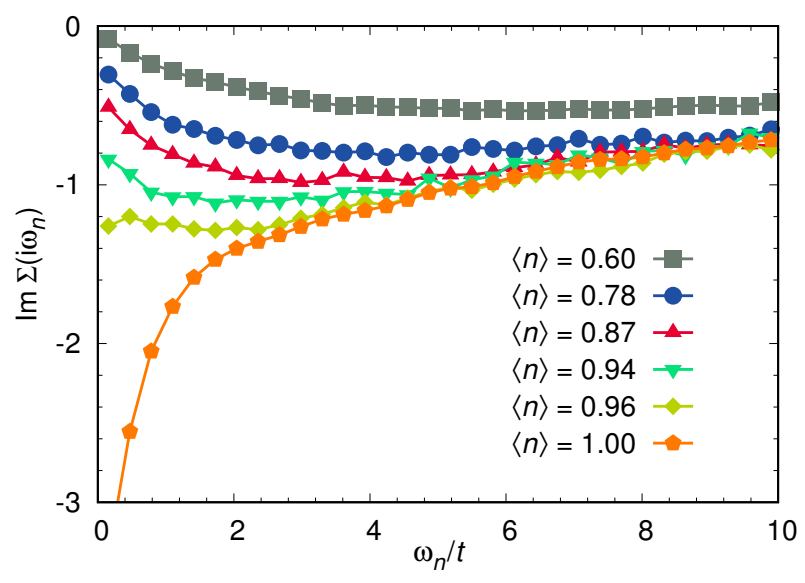

FIG. 7: (Color online) The imaginary part of on-site selfenergy $\operatorname{Im} \Sigma\left(\mathrm{i} \omega_{\mathrm{n}}\right)$ as a function of Matsubara frequency $\omega_{n}$ for $T / t=0.05, t_{\perp} / t=0.5$, and $U / t=6.0$ in different electron densities $\langle n\rangle$.

\section{CONCLUSIONS}

In conclusion, we investigated a two-dimensional bilayer Hubbard model on the square lattice as a function of band splitting and doping at a large interaction value $U / t=6.0$ by means of the dynamical cluster approximation with a $N_{c}=2 \times 4$ site cluster with short-range spatial as well as quantum fluctuations. The scattering rate, which indicates the degree of Mott physics, rapidly disappears in the momentum sectors $(\pi, 0)$ and $(0, \pi)$ with increasing interlayer hopping $t_{\perp} / t$. In fact we find a momentum-selective phase reentrant behavior from band insulating states at weak interlayer hopping $t_{\perp} / t \simeq 0.5$ to Mott insulating behavior at $t_{\perp} / t \simeq 2.0$ and then from Mott insulating to band insulating behavior at strong interlayer hopping $t_{\perp} / t \simeq 3$.0. These transitions are identified from the scattering rates in the $(\pi, \pi)$ momentum sector of the bonding band and the $(0,0)$ momentum sector of the anti-bonding band at half filling. Interesting phases are established with two consecutive phase transitions from a monolayer plaquette singlet Mott insulator (m-PSMI) to a band insulator through an intermediate phase, called bilayer plaquette singlet Mott insulator (bPSMI), where Mott physics is more present in the $(\pi, \pi)$ sector of the bonding band and the $(0,0)$ of the antibonding band, rather than in the $(\pi, 0)$ and $(0, \pi)$ sectors as usually observed in an antiferromagnetic Mott insulator. We attribute the unusual consecutive phases to competition and cooperation between short-range spatial correlations with quantum fluctuations and interlayer hopping $t_{\perp} / t$. The transition of Mott to band insulator with the absence of large scattering rates in all momentum sectors is found at large interlayer hopping $t_{\perp} / t \simeq 3.0$. Furthermore, since the momentum-dependent evolution of the insulating behavior with $t_{\perp} / t$ is strongly controlled by the evolution of the Fermi surface in the weak-coupling limit, we expect that consideration of larger clusters and 
different geometries in DCA won't change this scenario qualitatively.

Finally we also find that away from half filling, nonFermi liquid behavior is dominated by antiferromagnetic correlations rather than the finite scattering rate at the Fermi level. We suggest that this non-Fermi liquid behavior might be related to anomalous phenomena like the Fermi arc or hole pocket. We expect that momentumselective phenomena may exist in many cases which calls for further studies in various models and real materials where the short-range spatial fluctuations are emphasized.

\section{ACKNOWLEDGEMENT}

We would like to thank J. Kuneš, L. F. Tocchio, F. Becca, I.I. Mazin, M. Capone, and C. Gros for useful discussion. We gratefully acknowledge financial support from the Deutsche Forschungsgemeinschaft through grants FOR 1346 and SFB/TRR 49. Y.Z is supported by National Natural Science Foundation of China (No. 11174219), Shanghai Pujiang Program (No. 11PJ1409900), Research Fund for the Doctoral Program of Higher Education of China (No. 20110072110044) and the Program for Professor of Special Appointment (Eastern Scholar) at Shanghai Institutions of Higher Learning. H.L is supported by ERC/FP7 through the Starting Independent Grant "SUPERBAD", Grant Agreement No. 240524 .
* hplee@itp.uni-frankfurt.de

† yzzhang@tongji.edu.cn

1 N. F. Mott, Proceedings of the Physical Society of London Series A 62, 416 (1949).

2 F. Gebhard, The Mott Metal-Insulator Transition Springer Tracts in Modern Physics 137 Springer (1997).

3 M. Nakamura, Phys. Rev. B 61, 16377 (2000).

${ }^{4}$ Y. Z. Zhang, Phys. Rev. Lett. 92, 246404 (2004).

${ }^{5}$ K.-M. Tam, S.-W. Tsai, D. K. Campbell, Phys. Rev. Lett. 96, 036408 (2006).

6 S. Ejima, S. Nishimoto, Phys. Rev. Lett. 99, 216403 (2007).

7 M. Fabrizio, A. O. Gogolin, A. A. Nersesyan, Phys. Rev. Lett. 83, 2014 (1999).

8 Y. Z. Zhang, C. Q. Wu, H. Q. Lin, Phys. Rev. B 67, 205109 (2003).

9 C. D. Batista, A. A. Aligia, Phys. Rev. Lett. 92, 246405 (2004).

10 Ara Go, Gun Sang Jeon, Phys. Rev. B 84, 195102 (2011).

11 A. Garg, H. R. Krishnamurthy, M. Randeria, Phys. Rev. Lett. 97, 046403 (2006).

12 S. S. Kancharla, E. Dagotto, Phys. Rev. Lett. 98, 016402 (2007).

13 N. Paris, K. Bouadim, F. Hebert, G. G. Batrouni, R. T. Scalettar, Phys. Rev. Lett. 98, 046403 (2007).

14 H.-M. Chen, H. Zhao, H.-Q. Lin, C.-Q. W, New Journal of Physics 12, 093021 (2010).

15 L. Craco, P. Lombardo, R. Hayn, G. I. Japaridze, and E. Muller-Hartmann, Phys. Rev. B 78, 075121 (2008).

16 A. Damascelli, Z. Hussain, Z-X. Shen, Rev. Mod. Phys. 75, 473 (2003).

17 D. Fournier et al. Nature physics 6, 905 (2010).

18 A. Fuhrmann, D. Heilmann, H. Monien, Phys. Rev. B 73, 245118 (2006).

19 H. Hafermann, M. I. Katsnelson, A. I. Lichtenstein, Euro. Phys. Lett. 85, 37006 (2009).

20 S.S. Kancharla, S. Okamoto, Phys. Rev. B 75, 193103 (2007).

21 A. Georges, G. Kotliar, W. Krauth, M. J. Rozenberg, Rev.
Mod. Phys. 68, 13 (1996).

22 Y. Z. Zhang, M. Imada, Phys. Rev. B 76, 045108 (2007).

23 H. Park, K. Haule, G. Kotliar, Phys. Rev. Lett. 101, 186403 (2008).

24 E. Gull, P. Werner, W. Wang, M. Troyer, A. Millis, Europhys. Lett. 84, 37009 (2008).

25 H. Lee, Y.Z. Zhang, H.O. Jeschke, R. Valenti, H. Monien, Phys. Rev. Lett. 104, 026402 (2010).

26 T.A. Maier, D.J. Scalapino, Phys. Rev. B 84, 180513(R) (2011).

27 K. Bouadim, G.G. Batrouni, F. Hebert, R.T. Scalettar, Phys. Rev. B 77, 144527 (2008).

28 N. Lanata, P. Barone, M. Fabrizio, Phys. Rev. B 80, 224524 (2009).

29 M. H. Hettler, A. N. Tahvildar-Zadeh, M. Jarrell, T. Pruschke, and H. R. Krishnamurthy, Phys. Rev. B 58, 7475(R) (1998).

30 T. Maier, M. Jarrell, T. Pruschke, M.H. Hettler, Rev. Mod. Phys. 77, 1027 (2005)

31 A.N. Rubtsov, V.V. Savkin, A.I. Lichtenstein, Phys. Rev. B 72, 035122 (2005).

32 F.F. Assaad, T. C. Lang, Phys. Rev. B 76, 035116 (2007).

33 E. Gull, A. J. Millis, A. Lichtenstein, A. Rubtsov, M. Troyer, P. Werner, Rev. Mod. Phys. 83, 349 (2011).

34 Michael Sentef, Jan Kuneš, Philipp Werner, Arno P. Kampf, Phys. Rev. B 80, 155116 (2009).

35 for a comparison with other methods see L. F. Tocchio, H. Lee, H. O. Jeschke, R. Valenti, C. Gros Phys. Rev. B 87, 045111 (2013).

36 T. D. Stanescu, G. Kotliar, Phys. Rev. B 74, 125110 (2006).

37 S. Sakai, Y. Motome, M. Imada, Phys. Rev. Lett. 102, 056404 (2009).

38 E. Gull, M. Ferrero, O. Parcollet, A. Georges, A. J. Millis, Phys. Rev. B 82, 155101 (2010).

39 G. Sordi, K. Haule, A.-M.S. Tremblay, Phys. Rev. B 84, 075161 (2011).

40 S. Sakai, G. Sangiovanni, M. Civelli, Y. Motome, K. Held, M. Imada, Phys. Rev. B 85, 035102 (2012). 
${ }^{41}$ H. Lee, Y.-Z. Zhang, H.O. Jeschke, R. Valenti, Phys. Rev. B 84, 020401(R) (2011). 